\title{
Rationale for Neoadjuvant Chemotherapy of Resectable Colorectal Cancer Liver Metastases: When is it Useful?
}

\author{
Hiroto Kikuchi ${ }^{1,2, *}$, Shuichi Aoki ${ }^{1,3}$, Dan G. Duda ${ }^{1}$, Kohei Shigeta ${ }^{1,2}$
}

Corresponding author: Hiroto Kikuchi, MD, PhD

Edwin L. Steele Laboratories for Tumor Biology, Department of Radiation

Oncology, Massachusetts General Hospital, 100 Blossom St Cox-734

Boston, MA 02478, USA

Fax: 617-726-1962.

E-mail: HKIKUCHI2@mgh.harvard.edu
Received: 15.11.2019

Accepted: 05.01.2020

Copyright (c) Celsius Publishing House www.sgo-iasgo.com
'Edwin. L. Steele Laboratories for Tumor Biology, Department of Radiation Oncology, Massachusetts General Hospital and Harvard Medical School, Boston, MA, USA

${ }^{2}$ Department of Surgery, Keio University School of Medicine, Tokyo, Japan

${ }^{3}$ Department of Surgery, Tohoku University Graduate School of Medicine, Sendai, Japan

\begin{abstract}
Colorectal cancer is the third most common cancer and the third leading cause of cancer death in the United States (1). Moreover, approximately $20 \%$ of patients are diagnosed with synchronous colorectal liver metastases (CRLMs)(2). For patients with CRLM, the 5-year survival rate with after chemotherapy alone is approximately 11 percent (3). However, the 5 -year and 10-year survival rates after surgical resection are reported as $38 \%$ and $26 \%$, respectively (4). Therefore, surgery is considered a potentially curative intervention in CRLM. Unfortunately, not all CRLM patients are candidate for liver resection due to several factors. These include medical comorbidities and anatomical factors such as the number of metastases, tumor size and margin. In some cases, chemotherapy for unresectable tumors could convert the tumors to resectable. In other cases, neoadjuvant chemotherapy can help patients with synchronous metastatic disease undergo performed staged resection. Although neoadjuvant chemotherapy could be useful, its implementation is not yet standard and its use in resectable CRLM remains controversial. This review article focuses on the potential utility of neoadjuvant therapy for CRLMs.
\end{abstract}

Key words: colorectal cancer, liver metastases, neoadjuvant chemotherapy, resection, immune checkpoint blockade

\section{RATIONALE FOR LIVER METASTASES RESECTION}

\section{Which CRLMs are considered "resectable"?}

Surgical resection is widely regarded as the best intervention to cure and/or achieve long-term survival in CRLM patients. Since liver resection is major surgery, patients must be selected carefully by taking into consideration factors related to patients' fitness, to the tumors and to their anatomy. Comorbidities, such as other liver diseases, cardiovascular diseases and pulmonary diseases, age and past medical histories can be important risk factors. Factors related to the tumors include established prognostic scoring systems which also evaluate the benefit of neoadjuvant chemotherapy for CRLMs (5-12). The major compo- 
nents in these scoring systems are the size of the largest metastatic lesion, the number of metastases, margin, bilobar liver disease, $\mathrm{N}$ and $\mathrm{T}$ status, differentiation of primary tumor, CEA level, presence of resectable extrahepatic disease, and interval after primary or liver metastasis. Although few of these scoring systems could predict survival more than 5 years, these scoring are often used for treatment decisions (13). For patients with CRLM who require major liver resection, staged surgery with complementary procedures, such as the portal vein embolization (PVE) $(14,15)$ and the associating liver partition and portal vein ligation for staged hepatectomy (ALPPS) $(16,17)$, could be considered. Finally, regarding the anatomic factors, major limitations for resection could be the number of CRLMs, tumor size, and their location and margins. The continuous progress in the development of neoadjuvant chemotherapy and surgical procedure has simplified the criteria for resectability $(18,19)$.

\section{How about metachronous CRLMs?}

Metachronous CRLMs are liver metastases detected after curative surgery of a primary colorectal cancer. The surgical procedure for treating metachronous CRLM is largely similar to the one used for other liver tumors. If an $\mathrm{RO}$ resection is successfully performed for metachronous CRLMs, the 3-year survival and 5-year survival rates after liver resection are reported as $58 \%$ and $42 \%$, respectively (4). To estimate the efficacy of neoadjuvant chemotherapy over surgery first, Adam et al analyzed 1,471 patients with solitary and metachronous CRLMs; of these, 169 patients were treated with at least 3 cycles of an oxaliplatin - or irinotecan-based regimen before surgery while 1,302 patients received no preoperative chemotherapy (20). They reported that preoperative chemotherapy did not improve the disease-free survival (DFS) or overall survival (OS) in these patients with CRLMs of less than $5 \mathrm{~cm}$ in size. Thus, the benefit of neoadjuvant chemotherapy remains unclear if the metachronous CRLMs are few, small and easy to resect. However, if the metastatic lesions are multiple or bulky, neoadjuvant chemotherapy may be considered to reduce the burden and the risk of early recurrence after surgery for CRLM, as discussed below.

\section{What is the rationale for using neoadjuvant} chemotherapy?

Multiple chemotherapy regimens that include biologics have shown efficacy in metastatic colorectal cancer ( $\mathrm{mCRC}$ ) patients. Some of these regimens have been tested as neoadjuvant chemotherapies for CRLMs, their role and the optimal regimen are still unclear. Because of the potential resistance and/or toxicity to these drugs, the benefit for neoadjuvant chemotherapy for patients with low risk, few and small tumors is questionable. On the other hand, for patients with high risk, unresectable or borderline resectable, neoadjuvant chemotherapy is considered as an appropriate option. Once neoadjuvant chemotherapy is initiated, it is recommended that the CRLM patients are followed by radiographic examination every 6-8 weeks. To evaluate the effect of the regimen, the RECIST criteria are commonly used (21). CRLMs resection should be performed as soon as possible after tumors are deemed resectable, but at least 4 weeks after finishing chemotherapy (or even longer if the antiangiogenic drug bevacizumab is used) to avoid surgical complications.

\section{STANDARD REGIMENS FOR METASTATIC COLORECTAL CANCER}

The regimens that have shown efficacy in randomized phase III clinical trials for $\mathrm{MCRC}$ are multiple and include doublet or triplet chemotherapies with biologics. Fluorouracil (FU) and leucovorin (LV) are being combined with oxaliplatin or irinotecan or both, regimens referred to as FOLFOX $(22)$, FOLFIRI $(23,24)$ and FOLFOXIRI (25-27), respectively. There are alsomodified options for these regimens (using oral capecitabine $(28,29)$ or S-1 $(30,31)$ instead of FU and LV) that have also shown efficacy in randomized trials. In addition, several molecularly targeted drugs have shown efficacy. These include antiangiogenic (anti-vascular endothelial growth factor (VEGF) pathway) drugs such bevacizumab (antiVEGF antibody)(32-34), aflibercept (chimeric soluble VEGF receptor (VEGFR)-1 or "VEGF-trap")(35), ramucirumab (anti-VEGFR2 antibody)(36) and regorafenib (multikinase inhibitor of VEGFRs and RAF)(37), or tumor-targeted drugs such asanti-epithelial growth factor receptor (EGFR) antibodies (cetuximab and panitumumab) (38-40). Cetuximab and panitumumab are only used for patients with wild-type RAS/BRAF status, because these tumors are invariably resistant to EGFR blockade. Moreover, an analysis of six randomized trials of EGFR antibodies showed a better outcome of this intervention for patients with left-sided CRCs than right-sided CRCs (41). This study showed that tumor sidedness could be one of the critical factors for selecting treatment regimens. TAS-102, an oral drug consisting of trifluridine and tipiracil hydrochloride, has also become a treatment 
option for mCRC (42). Finally, while immunotherapy with immune checkpoint blockers has failed to show substantial activity so far in $\mathrm{MCRC}$, blockade of programmed cell death receptor (PD)-1 and cytotoxic $T$ lymphocyte associated protein (CTLA)- 4 may be an efficacious option for CRC patients with tumors with deficiencies in mismatch repair (dMMR) or high levels of microsatellite instability (MSI-H); this includes the anti-PD-1 antibody nivolumab with or without the antiCTLA-4 antibody ipilimumab (43). In this study, overall response rate was $55 \%$, and progression-free survival rates were $76 \%$ at 9 months and $71 \%$ at 1 year. The promising data from the limited experience with immune checkpoint inhibitors in $\mathrm{MCRC}$ needs to be validated in larger trials, and its role in other stages of the disease (for example in neoadjuvant setting) remains to be established.

\section{LIVER TOXICITIES CAUSED BY CHEMOTHERAPY OR BIOLOGICS}

In considering neoadjuvant treatment for CRLM, an important factor is the characteristic liver toxicity of the drugs, which could pose a risk for liver resection (44, 45). Oxaliplatin causes sinusoidal obstruction syndrome, which is similar to a venous occlusive disease. Noncirrhotic portal hypertension has also been reported for the use of oxaliplatin with fluorouracil (45). Irinotecan can cause steatosis and steatohepatitis - adverse effects which correlate with highmortality (46). A more recent report onliver toxicity after preoperative chemotherapy shows that steatohepatitis was observed more frequently after irinotecan than after oxaliplatin (14.8\% vs $3.4 \%)$ and in patients with body mass index $(\mathrm{BMI})>25 \mathrm{~kg} / \mathrm{m}^{2}(\mathrm{OR}=10.0)(47)$. Another paper shows that the preoperative aspartate aminotransferase-to-platelet ratio index can predict liverrelated complications after neoadjuvant chemotherapy and is related to sinusoidal obstruction syndrome (48). Bevacizumab is often used with oxaliplatin or irinotecan regimen. However, bevacizumab can cause adverse effects such as thromboembolic disease, bleeding and bowel perforation. In addition, it may also cause delays in wound and liver healing post-surgery (49). Therefore, liver resection is usually planned 6 to 8 weeks after bevacizumab therapy, given its prolonged half-life in blood circulation.

\section{GUIDELINES FOR INITIALLY RESECTABLE CRLMS}

In the United States of America, the guidelines set by the National Comprehensive Cancer Network (NCCN) recommended upfront surgery if the tumors are initially resectable (50). For cases where there are more than 4 metastases, suspicion of portal node metastasis or bilobar disease, neoadjuvant chemotherapy is preferred prior to surgical resection. Once neoadjuvant chemotherapy starts, it is critically important to check the tumor status every 6 to 8 weeks using radiographic examinations to minimize the number of chemotherapy courses. In some cases, the CRLMs recur in remaining liver rapidly after resection. Most likely, these CRLMs are present at the time of resection and are particularly aggressive. For these cases, two or three cycles of preoperative chemotherapy may be a useful option to determine whether these CRLM patients will derive benefit from surgical intervention or not (51). In the largest study performed to date, the European Organization for Research and Treatment of Cancer (EORTC) investigators tested whether perioperative chemotherapy can improve survival in CRLM patients over surgery alone (EORTC Intergroup trial 40983). Patients were randomly assigned to receive FOLFOX4 versus surgery upfront $(52,53)$. In this study, 12 weeks of chemotherapy was planned for both preand post-operative period. Sixty-seven of 182 patients were included and about $80 \%$ of them were resected in both groups. The HR for progression-free survival was $0.79(p=0.058)$ in all randomly assigned patients; this corresponded to an increase in median progressionfree survival from 11.7 months to 18.7 months with the addition of chemotherapy. This difference was significant in the patients in whom resection was actually achieved after study entry (HR was 0.73, $\mathrm{p}=0.025$ ). However, after 8.5 years of follow up, there was no significant statistical differences in 5 -year overall survival $(52,53)$. Of note, the trial was not powered to detect a difference in overall survival. The study investigators concluded that - while the difference in survival shown in this study was not statistically significant - further evaluation of perioperative chemotherapy with FOLFOX4 (with or without biologics) is warranted for resectable CRLM. In conclusion, the benefit of neoadjuvant chemotherapy remains controversial for initially resectable CRLMs.

\section{SYSTEMIC NEOADJUVANT THERAPY REGIMENS FOR INITIALLY RESECTABLE CRLMS}

Recently, the number of drugs and regimens that showed efficacy for mCRChas been rapidly increasing. The suggestions included in the NCCN and European 
Society for Medical Oncology (ESMO) guidelines for initially resectable CRLMs include the use of FOLFOX, FOLFIRI, XELOX (capecitabine and oxaliplatin) with or without bevacizumab, and FOLFIRI, FOLFOX with or without cetuximab or panitumumab (54). More recently, FOLFOXIRI was also added in the NCCN guideline as a treatment option for neoadjuvant therapy. Adding bevacizumab to the 5-FU and oxaliplatin regimen may improve pathological responses and reduce liver injury (55). Similarly, adding anti-EGFR antibody to chemotherapies can produce "early tumor shrinkage", and this effect is associated with superior long-term outcomes (56). While these regimens might be useful, there are some critical points that must be kept in mind for choosing regimens. For the use of antiVEGF as part of neoadjuvant treatment for patients with resectable CRLMs, the concerns are the potential adverse effects of bevacizumab (49). For the use of anti-EGFR therapy, the concerns is the efficacy of the intervention, as the result of the EPOC trial which showed no difference in OS and an inferior progression-free survival (PFS) when cetuximab was added to FOLFOX $(57,58)$.

\section{Potential systemic therapy regimens for unresectable CRLMs}

For patients with initially unresectable CRLMs, there is strong rationale for preoperative chemotherapy. Usually patients with initially un-resectable CRLMs receive at least several courses of chemotherapy to evaluate tumor response and reevaluate the resectability. Longer interventions may cause liver damage, which in turn may cause postoperative complications. If neoadjuvant chemotherapy is effective and converts the unresectable CRLMs to resectable, an immediate liver resection is recommended. There ported success rates for conversion after neoadjuvant therapy range between $12-33 \%(25,59-66)$. The reported 5-year survival rate after resection are $30-54 \%$, which is considerably higher than that after chemotherapy alone (approximately 10\%). After chemotherapy, the rate of complete pathologic response is reported at $4-9 \%$ $(60,67,68)$. Even if CRLMs show complete radiologic response after chemotherapy, the vast majority of cases showed persistent macroscopic or microscopic residual disease or early recurrence in situ (69). Thus, resection is strongly indicated even if the CRLMs show complete response by imaging. In these case, identifying tumor locations is critically important; an option to address this problem is using a marking technique consisting of placing coils using computed tomography or ultrasound guidance before chemotherapy (70).

For initially unresectable CRLMs, all the regimens for unresectable mCRCs are considered appropriate. However, there is no standard regimen for this particular setting. If conversion from initially unresectable or borderline to resectable is expected, there are some recommendations. These include FOLFOX or FOLFOXIRI; for patients with-wild type of RAS and BRAF, FOLFIRI plus cetuximab or panitumumab could also be considered. Since potent cytotoxic regimensare preferred for attempting conversion, doublet therapies which contain either oxaliplatin or irinotecan are usually selected; FOLFOX is more commonly selected than FOLFIRI because irinotecan may cause steatohepatitis. There are some early reports on the use of FOLFOXIRI, which contains both oxaliplatin and irinotecan and appears to be useful for young patients or patients without comorbidities $(25,64)$. From the retrospective analysis of the TRIBE trial, FOLFOXIRI plus bevacizumab may be one of the options for the first-line regimen for patients with right-sided mCRCs regardless of their status of RAS or BRAF mutation, because of their worse prognosis compared to left-sided tumors (71). Finally, while the use of immune checkpoint inhibitors is now standard for unresectable mCRCs with dMMR MSI-H tumors, their safety and utility in neoadjuvant setting remains to be examined.

\section{CONCLUSION}

Surgical resection for CRLMs remains the intervention of choice to achieve cure in this advanced disease setting. Although a recent meta-analysis including 18 studies $(6,254$ patients) shows that neoadjuvant chemo-therapy can improve both 5-year DFS (HR 1.38) and 5 -year OS $(H R=1.19)(72)$, there are no currently selection criteria for patients and no prospective evidence to prove the benefit of combining targeted agents such as cetuximab and bevacizumab (73). Therefore, for initially resectable CRLMs, resection is usually recommended if feasible. For initially unresectable CRLMs, chemotherapy using cytotoxic regimens should be considered to convert the tumors to resectable. With the advent of multiple effective cytotoxic regimens, biologics and immune checkpoint inhibitors for $\mathrm{mCRCs}$, there are great expectations that new systemic neoadjuvant therapy will further improve the survival outcomes. However, the safety and efficacy of these interventions should be tested and validated in future randomized studies. 


\section{Conflict of interest}

The authors declare no conflict of interest with this work.

\section{REFERENCES}

1. Cronin KA, Lake AJ, Scott S, Sherman RL, Noone AM, Howlader N, et al. Annual Report to the Nation on the Status of Cancer, part I: National cancer statistics. Cancer. 2018;124(13): 2785-2800.

2. Leporrier J, Maurel J, Chiche L, Bara S, Segol P, Launoy G. A population-based study of the incidence, management and prognosis of hepatic metastases from colorectal cancer. Br J Surg. 2006;93(4):465-74.

3. Ferrarotto R, Pathak P, Maru D, Agarwal A, Overman M, Hoff PM, et al. Durable complete responses in metastatic colorectal cancer treated with chemotherapy alone. Clin Colorectal Cancer. 2011 10(3):178-82.

4. Kanas GP, Taylor A, Primrose JN, Langeberg WJ, Kelsh MA, Mowat $\mathrm{FS}$, et al. Survival after liver resection in metastatic colorectal cancer: review and meta-analysis of prognostic factors. Clin Epidemiol. 2012;4:283-301.

5. Konopke R, Kersting S, Distler M, Dietrich J, Gastmeier J, Heller A, et al. Prognostic factors and evaluation of a clinical score for predicting survival after resection of colorectal liver metastases. Liver Int. 2009;29(1):89-102.

6. Nagashima I TT, Adachi M, Nagawa H, Muto T, and O. K. Proposal of criteria to select candidates with colorectal liver metastases for hepatic resection: Comparison of our scoring system to the positive number of risk factors. World J Gastroenterol. 2006 12(39):6305-6309.

7. Nordlinger B GM, Vaillant JC, Balladur P, Boudjema K, Bachellier P Jaeck D. Surgical resection of colorectal carcinoma metastases to the liver. A prognostic scoring system to improve case selection, based on 1568 patients. Association Française de Chirurgie. Cancer, 1996;77(7):1254-62.

8. Rees M, Tekkis PP, Welsh F K S, O'Rourke T, John TG. Evaluation of long-term survival after hepatic resection for metastatic colorectal cancer: a multifactorial model of 929 patients. Ann Surg. 2008 247(1):125-35

9. Shunzaburo Iwatsuki ID, Madariaga JR, Wallis Marsh J, Dodson F, Bonham AC, Geller DA, et al. Hepatic Resection for Metastatic Colorectal Adenocarcinoma: A Proposal of a Prognostic Scoring System. J Am Coll Surg. 1999:189(3):291-299.

10. Yuman Fong M, Fortner J, Sun RL, Brennan MF, Blumgart LH. Clinical Score for Predicting Recurrence After Hepatic Resection for Metastatic Colorectal Cancer Analysis of 1001 Consecutive Cases. Annals of Surgery.1999;230(3):309-321.

11. Zakaria S, Donohue JH, Que FG, Farnell MB, Schleck CD, Ilstrup DM et al. Hepatic resection for colorectal metastases: value for risk scoring systems? Ann Surg. 2007;246(2):183-91.

12. Beppu T, Sakamoto $Y$, Hasegawa $K$, Honda G, Tanaka K, Kotera $Y$, et al. A nomogram predicting disease-free survival in patients with colorectal liver metastases treated with hepatic resection: multicenter data collection as a Project Study for Hepatic Surgery of the Japanese Society of Hepato-Biliary-Pancreatic Surgery. J Hepatobiliary Pancreat Sci. 2012;19(1):72-84.

13. Roberts KJ, White A, Cockbain A, Hodson J, Hidalgo E, Toogood GJ, Lodge JPA. Performance of prognostic scores in predicting longterm outcome following resection of colorectal liver metastases. $\mathrm{Br}$ J Surg. 2014;101(7):856-66.

14. Aoki T, Kubota K. Preoperative portal vein embolization for hepatocellular carcinoma: Consensus and controversy. World J Hepatol. 2016;8(9):439-45

15. Makuuchi M TK, Takuma T, Yamazaki S, Hasegawa H, Nishimura S Shimamura Y. Preoperative transcatheter embolization of the portal venous branch for patients receiving extended lobectomy due to the bile duct carcinoma. J Jpn Soc Clin Surg. 1984;45:14-20.
16. de Santibanes E, Clavien PA. Playing Play-Doh to prevent postoperative liver failure: the "ALPPS" approach. Ann Surg. 2012; 255(3):415-7.

17. Schadde E, Schnitzbauer AA, Tschuor C, Raptis DA, Bechstein WO, Clavien PA et al. Systematic review and meta-analysis of feasibility, safety, and efficacy of a novel procedure: associating liver partition and portal vein ligation for staged hepatectomy. Ann Surg Oncol. 2015;22(9):3109-20

18. Khatri VP, Petrelli NJ, Belghiti J. Extending the frontiers of surgical therapy for hepatic colorectal metastases: is there a limit? J Clin Oncol. 2005;23(33):8490-9.

19. Berri RN, a.E.K.A. Curable Metastatic Colorectal Cancer: Recommended Paradigms. Curr Oncol Rep. 2009;11:200-208.

20. Adam R, Bhangui P, Poston G, Mirza D, Nuzzo G, Barroso E, et al. Is perioperative chemotherapy useful for solitary, metachronous, colorectal liver metastases? Ann Surg. 2010;252(5):774-87.

21. Eisenhauer EA, Therasse P, Bogaerts J, Schwartz LH, Sargent D, Ford R, Dancey J, et al. New response evaluation criteria in solid tumours: revised RECIST guideline (version 1.1). Eur J Cancer. 2009:45(2):228-47.

22. Goldberg RM, Sargent DJ, Morton RF, Fuchs CS, Ramanathan RK, Williamson SK, et al. A randomized controlled trial of fluorouracil plus leucovorin, irinotecan, and oxaliplatin combinations in patients with previously untreated metastatic colorectal cancer. J Clin Oncol, 2004;22(1):23-30

23. Saltz LB, Cox JV, Blanke C, Rosen LS, Fehrenbacher L, Moore MJ, et al. Irinotecan plus fluorouracil and leucovorin for metastatic colorectal cancer. Irinotecan Study Group. N Engl J Med. 2000;343(13): 905-914.

24. Douillard JY, Cunningham D, Roth AD, Navarro M, James RD, Karasek $\mathrm{P}$, et al. Irinotecan combined with fluorouracil compared with fluorouracil alone as first-line treatment for metastatic colorectal cancer: a multicentre randomised trial. Lancet. 2000; 355(9209):1041-1047.

25. Falcone A, Ricci S, Brunetti I, Pfanner E, Giacomo A, Barbara C, et al. Phase III trial of infusional fluorouracil, leucovorin, oxaliplatin, and irinotecan (FOLFOXIRI) compared with infusional fluorouracil, leucovorin, and irinotecan (FOLFIRI) as first-line treatment for metastatic colorectal cancer: the Gruppo Oncologico Nord Ovest. J Clin Oncol. 2007;25(13):1670-6.

26. Loupakis F, Cremolini C, Masi G, Lonardi S, Zagonel V, Salvatore L, et al. Initial therapy with FOLFOXIRI and bevacizumab for metastatic colorectal cancer. N Engl J Med. 2014;371(17):1609-18.

27. Cremolini C, Loupakis F, Antoniotti C, Lupi C, Sensi E, Lonardi S, et al. FOLFOXIRI plus bevacizumab versus FOLFIRI plus bevacizumab as first-line treatment of patients with metastatic colorectal cancer: updated overall survival and molecular subgroup analyses of the open-label, phase 3 TRIBE study. Lancet Oncology. 2015;16(13): 1306-1315.

28. Van Cutsem E, Twelves C, Cassidy J, Allman D, Bajetta E, Boyer M, et al. Oral Capecitabine Compared With Intravenous Fluorouracil Plus Leucovorin in Patients With Metastatic Colorectal Cancer: Results of a Large Phase III Study. J Clin Oncol. 2001;19(21): 4097-4106

29. Cunningham D, Lang I, Marcuello E, Lorusso V, Ocvirk J, Bok Shin $D$, et al. Bevacizumab plus capecitabine versus capecitabine alone in elderly patients with previously untreated metastatic colorectal cancer (AVEX): an open-label, randomised phase 3 trial. Lancet Oncology. 2013;14(11):1077-1085.

30. Muro K, Boku N, Shimada Y, Tsuji A, Sameshima S, Baba H, et al. Irinotecan plus S-1 (IRIS) versus fluorouracil and folinic acid plus irinotecan (FOLFIRI) as second-line chemotherapy for metastatic colorectal cancer: a randomised phase $2 / 3$ non-inferiority study (FIRIS study). Lancet Oncology. 2010;11(9):853-860.

31. Komatsu Y, Yuki S, Sogabe S, Fukushima H, Iwanaga I, Kudo M, et al. Phase II study of combined treatment with irinotecan and S-1 (IRIS) in patients with inoperable or recurrent advanced colorectal cancer (HGCSG0302). Oncology. 2011;80(1-2): 70-5.

32. Hurwitz H, Novotny W, Cartwright T, Hainsworth J, Heim W, Berlin J, et al. Bevacizumab plus Irinotecan, Fluorouracil, and Leucovorin for Metastatic Colorectal Cancer. The new england journal of medicine. 
2004:350(23):2335-2342.

33. Saltz LB, Clarke S, Díaz-Rubio E, Scheithauer W, Figer A, Wong R, et al. Bevacizumab in combination with oxaliplatin-based chemotherapy as first-line therapy in metastatic colorectal cancer: a randomized phase III study. J Clin Oncol. 2008;26(12):2013-9.

34. Fuchs CS, Marshall J, Barrueco J. Randomized, controlled trial of irinotecan plus infusional, bolus, or oral fluoropyrimidines in first-line treatment of metastatic colorectal cancer: updated results from the BICC-C study. J Clin Oncol. 2008;26(4):689-90.

35. Van Cutsem E, Tabernero J, Lakomy R, Prenen H, Prausová J, Macarulla T, et al. Addition of aflibercept to fluorouracil, leucovorin, and irinotecan improves survival in a phase III randomized trial in patients with metastatic colorectal cancer previously treated with an oxaliplatin-based regimen. J Clin Oncol. 2012;30(28):3499-506.

36. Tabernero J, Yoshino T, Lee Cohn A, Obermannova R, Bodoky G, Garcia-Carbonero $R$, et al. Ramucirumab versus placebo in combination with second-line FOLFIRI in patients with metastatic colorectal carcinoma that progressed during or after first-line therapy with bevacizumab, oxaliplatin, and a fluoropyrimidine (RAISE): a randomised, double-blind, multicentre, phase 3 study. Lancet Oncology. 2015;16(5):499-508.

37. Grothey A, Van Cutsem E, Sobrero A, Siena S, Falcone A, Ychou M, et al. Regorafenib monotherapy for previously treated metastatic colorectal cancer (CORRECT): an international, multicentre, randomised, placebo-controlled, phase 3 trial. Lancet. 2013; 381(9863):303-312.

38. Bokemeyer C, Bondarenko I, Makhson A, Hartmann JT, Aparicio J, de Braud $F$, et al. Fluorouracil, leucovorin, and oxaliplatin with and without cetuximab in the first-line treatment of metastatic colorectal cancer. J Clin Oncol. 2009;27(5):663-71.

39. Van Cutsem E, Köhne CH, Hitre E, Zaluski J, Chang Chien CR, Makhson A, et al. Cetuximab and Chemotherapy as Initial Treatment for Metastatic Colorectal Cancer. N Engl J Med. 2009;360(14): 1408-1417.

40. Douillard JY, Siena S, Cassidy J, Tabernero J, Burkes R, Barugel M, et al. Randomized, phase III trial of panitumumab with infusional fluorouracil, leucovorin, and oxaliplatin (FOLFOX4) versus FOLFOX4 alone as first-line treatment in patients with previously untreated metastatic colorectal cancer: the PRIME study. J Clin Oncol. 2010; 28(31):4697-705.

41. Arnold D, Lueza B, Douillard J-Y, Peeters M, Lenz H-J, Venook A, et al. Prognostic and predictive value of primary tumour side in patients with RAS wild-type metastatic colorectal cancer treated with chemotherapy and EGFR directed antibodies in six randomized trials. Ann Oncol. 2017;28(8):1713-1729.

42. Mayer RJ, Van Cutsem E, Falcone A, Yoshino T, Garcia-Carbonero R, Mizunuma N, et al. Randomized trial of TAS-102 for refractory metastatic colorectal cancer. N Engl J Med. 2015;372(20):1909-19.

43. Overman MJ, Lonardi S, Wong KYM, Lenz HJ, Gelsomino F, Aglietta $M$, et al. Durable clinical benefit with nivolumab plus Ipilimumab in DNA Mismatch Repair-Deficient/Microsatellite Instability-High Metastatic Colorectal Cancer. J Clin Oncol. 2018;36:773-779.

44. Robinson SM, Wilson CH, Burt AD, Manas DM, Steven A White et al. Chemotherapy-associated liver injury in patients with colorectal liver metastases: a systematic review and meta-analysis. Ann Surg Oncol. 2012;19(13):4287-99.

45. Zhao J, van Mierlo KMC, Gómez-Ramírez J, Kim H, Pilgrim CHC, Pessaux $P$, et al. Systematic review of the influence of chemotherapy-associated liver injury on outcome after partial hepatectomy for colorectal liver metastases. Br J Surg. 2017;104(8):990-1002.

46. Vauthey JN, Pawlik TM, Ribero D, Wu TT, Zorzi D, Hoff PM, et al. Chemotherapy regimen predicts steatohepatitis and an increase in 90-day mortality after surgery for hepatic colorectal metastases. J Clin Oncol. 2006;24(13):2065-72.

47. Desjardin M, Bonhomme B, Le Bail B, Evrard S, Brouste V, Desolneux $\mathrm{G}$, et al. Hepatotoxicities induced by neoadjuvant chemotherapy in colorectal cancer liver metastases: distinguishing the true from the false. Clin Med Insights Oncol. 2019;13 1179554918825450 .

48. Amptoulach S, Gross G, Sturesson C, Rissler P, Kalaitzakis E. Preoperative aspartate aminotransferase-to-platelet ratio index predicts perioperative liver-related complications following liver resection for colorectal cancer metastases. Scand J Surg. 2017; 106(4):311-317.

49. Scappaticci FA, Fehrenbacher L, Cartwright T, Hainsworth JD, Heim W, Berlin J, et al. Surgical wound healing complications in metastatic colorectal cancer patients treated with bevacizumab. J Surg Oncol. 2005;91(3):173-80.

50. Benson AB, Venook AP, Al-Hawary M M, Cederquist L, Chen YJ, Ciombor KK, et al. NCCN Guidelines Insights: Colon Cancer, Version 2.2018. J Natl Compr Canc Netw. 2018;16(4):359-369.

51. Kopetz, S. and J.-N. Vauthey, Perioperative chemotherapy for resectable hepatic metastases. The Lancet, 2008. 371(9617): p. 963-965.

52. Nordlinger B, Sorbye H, Glimelius B, Poston GJ, Schlag PM, Rougier $P$, et al. Perioperative chemotherapy with FOLFOX4 and surgery versus surgery alone for resectable liver metastases from colorectal cancer (EORTC Intergroup trial 40983): a randomised controlled trial. Lancet. 2008:371(9617):1007-1016.

53. Nordlinger B, Sorbye H, Glimelius B, Poston GJ, Schlag PM, Rougier $\mathrm{P}$, et al. Perioperative FOLFOX4 chemotherapy and surgery versus surgery alone for resectable liver metastases from colorectal cancer (EORTC 40983): long-term results of a randomised, controlled, phase 3 trial. Lancet Oncol. 2013;14(12):1208-1215.

54. Van Cutsem E, Cervantes A, Adam R, Sobrero A, Van Krieken JH, Aderka D, et al. ESMO consensus guidelines for the management of patients with metastatic colorectal cancer. Ann Oncol. 2016;27(8): 1386-422.

55. Ribero D, Wang H, Donadon M, Zorzi D, Thomas MB, Eng C, et al. Bevacizumab improves pathologic response and protects against hepatic injury in patients treated with oxaliplatin-based chemotherapy for colorectal liver metastases. Cancer. 2007;110(12): 2761-7.

56. Piessevaux $\mathrm{H}$, Buyse $\mathrm{M}$, Schlichting $\mathrm{M}$, Van Cutsem E, Bokemeyer $\mathrm{C}$, Heeger S, et al. Use of early tumor shrinkage to predict long-term outcome in metastatic colorectal cancer treated with cetuximab. J Clin Oncol. 2013;31(30):3764-75.

57. Primrose J, Falk S, Finch-Jones M, Valle J, O'Reilly D, Siriwardena $A$, et al. Systemic chemotherapy with or without cetuximab in patients with resectable colorectal liver metastasis: the New EPOC randomised controlled trial. Lancet Oncol. 2014.15(6):601-611.

58. Siân Pugh RT, Bridgewater J, Grisoni ML, Moutasim K, Rousseau F, Thomas GJ, et al. Association between miR-31-3p expression and cetuximab efficacy in patients with KRAS wild-type metastatic colorectal cancer: a post-hoc analysis of the New EPOC trial. Oncotarget. 2017;8:93856-93866

59. Wein A, CR, Kockerling F, Martus P, Baum U, Brueckl WM, Reck T, et al. Impact of surgery on survival in palliative patients with metastatic colorectal cancer after first line treatment with weekly 24-hour infusion of high dose 5 -fluorouraeil and folinic acid. Annals of Oncology. 2001;12:1721-1727.

60. Adam R, Wicherts DA, de Haas RJ, Ciacio O, Lévi F, Paule B, et al. Patients with initially unresectable colorectal liver metastases: is there a possibility of cure? J Clin Oncol. 2009;27(11):1829-35.

61. Alberts SR, Horvath WL, Sternfeld WC, Goldberg RM, Mahoney MR, Dakhil SR, et al. Oxaliplatin, fluorouracil, and leucovorin for patients with unresectable liver-only metastases from colorectal cancer: a North Central Cancer Treatment Group phase II study. J Clin Oncol. 2005;23(36):9243-9

62. Barone C, Nuzzo G, Cassano A, Basso M, Schinzari G, Giuliante F et al. Final analysis of colorectal cancer patients treated with irinotecan and 5-fluorouracil plus folinic acid neoadjuvant chemotherapy for unresectable liver metastases. Br J Cancer. 2007;97(8):1035-9.

63. Delaunoit T, Alberts SR, Sargent DJ, Green E, Goldberg RM, Krook $J$, et al. Chemotherapy permits resection of metastatic colorectal cancer: experience from Intergroup N9741. Ann Oncol. 2005;16(3): 425-9.

64. Masi G, Loupakis F, Pollina L, Vasile E, Cupini S, Ricci S, et al. Longterm outcome of initially unresectable metastatic colorectal cancer patients treated with 5-fluorouracil/leucovorin, oxaliplatin, and irinotecan (FOLFOXIRI) followed by radical surgery of metastases. Ann Surg. 2009;249(3):420-5. 
65. Ychou M, Viret F, Kramar A, Desseigne F, Mitry E, Guimbaud R, et al. Tritherapy with fluorouraci//leucovorin, irinotecan and oxaliplatin (FOLFIRINOX): a phase II study in colorectal cancer patients with non-resectable liver metastases. Cancer Chemother Pharmacol. 2008;62(2):195-201.

66. Beppu T, Miyamoto Y, Sakamoto Y, Imai K, Nitta H, Hayashi H, et al. Chemotherapy and targeted therapy for patients with initially unresectable colorectal liver metastases, focusing on conversion hepatectomy and long-term survival. Ann Surg Oncol. 2014;21 Suppl 3:S405-13.

67. Adam R, Wicherts DA, de Haas RJ, Aloia T, Lévi F, Paule B, et al. Complete pathologic response after preoperative chemotherapy for colorectal liver metastases: myth or reality? J Clin Oncol. 2008 26(10): 1635-41.

68. Blazer DG 3rd, Kishi Y, Maru DM, Kopetz S, Shin Chun Y, Overman $\mathrm{MJ}$, et al. Pathologic response to preoperative chemotherapy: a new outcome end point after resection of hepatic colorectal metastases. J Clin Oncol. 2008;26(33):5344-51.

69. Benoist S, Brouquet A, Penna C, Julié C, El Hajjam M, Chagnon
$S$, et al. Complete response of colorectal liver metastases after chemo-therapy: does it mean cure? J Clin Oncol. 2006;24(24): 3939-45.

70. Zalinski S, Abdalla EK, Mahvash A, Vauthey JN. A marking technique for intraoperative localization of small liver metastases before systemic chemotherapy. Ann Surg Oncol. 2009;16(5): 1208-11.

71. Cremolini C, Antoniotti C, Lonardi S, Bergamo F, Cortesi E, Tomasello $\mathrm{G}$, et al. Primary tumor sidedness and benefit from FOLFOXIRI plus bevacizumab as initial therapy for metastatic colorectal cancer. Retrospective analysis of the TRIBE trial by GONO. Ann Oncol. 2018;29(7):1528-2534.

72. Liu W, Zhou JG, Sun $Y$, Zhang L, Xing BC, et al. The role of neoadjuvant chemotherapy for resectable colorectal liver metastases: a systematic review and meta-analysis. Oncotarget. 2016; 7(24):37277-37287.

73. Ke S, Zhan S, Zhu H, Yan D. Topics related to neoadjuvant chemotherapy for resectable liver metastases from colorectal cancer. J Buon. 2018;23(2):296-301. 